\title{
Dexmedetomidine hydrochloride as a long-term sedative
}

\author{
This article was published in the following Dove Press journal: \\ Therapeutics and Clinical Risk Management \\ II July 20 I I \\ Number of times this article has been viewed
}

\author{
Takayuki Kunisawa \\ Surgical Operation Department, \\ Asahikawa Medical University \\ Hospital, Asahikawa, Hokkaido, Japan
}

\begin{abstract}
Dexmedetomidine undoubtedly is a useful sedative in the intensive care setting because it has a minimal effect on the respiratory system. Dexmedetomidine infusions lasting more than 24 hours have not been approved since the first approval was acquired in the US in 1999. However, in 2008, dexmedetomidine infusions for prolonged use were approved in Colombia and in the Dominican Republic, and the number of countries that have granted approval for prolonged use has been increasing every year. This review discusses the literature examining prolonged use of dexmedetomidine and confirms the efficacy and safety of dexmedetomidine when it is used for more than 24 hours. Dexmedetomidine was administered at varying doses $(0.1-2.5 \mu \mathrm{g} / \mathrm{kg} /$ hour $)$ and durations up to 30 days. Dexmedetomidine seems to be an alternative to benzodiazepines or propofol for achieving sedation in adults because the incidences of delirium and coma associated with dexmedetomidine are lower than the corresponding incidences associated with benzodiazepines and propofol, although dexmedetomidine administration can cause mild adverse effects such as bradycardia. Controlled comparative studies on the efficacy and safety of dexmedetomidine and other sedatives in pediatric patients have not been reported. However, dexmedetomidine seems to be effective in managing extubation, reducing the use of conventional sedatives, and as an alternative for inducing sedation in patients for whom traditional sedatives induce inadequate sedation. Prolonged dexmedetomidine infusion has not been reported to have any serious adverse effects. Dexmedetomidine appears to be an alternative long-term sedative, but further studies are needed to establish its efficacy and safety.
\end{abstract}

Keywords: dexmedetomidine, prolonged sedation, long-term

\section{Introduction}

Dexmedetomidine is a highly selective $\alpha_{2}$-agonist with sedative, analgesic, and sympatholytic properties. ${ }^{1,2}$ Because of its minimal effects on the respiratory system, dexmedetomidine seems to be an ideal sedative. However, dexmedetomidine has not been widely used because its indications are limited to treatment of patients who are intubated, mechanically ventilated, and admitted to an intensive care unit. In addition, infusion cannot be continued beyond 24 hours, and the maximum recommended dose is only $0.7 \mu \mathrm{g} / \mathrm{kg} / \mathrm{hour}$.

Fortunately, the indications for the use of dexmedetomidine have been expanding, and the impetus for this change may have come from clinicians. Tables 1 and 2 show the approval status for dexmedetomidine in various settings in some countries. In October 2008, the US Food and Drug Administration (FDA) approved the use of dexmedetomidine in nonintubated patients prior to and during surgical and other procedures. Furthermore, the approved range of dexmedetomidine titration in monitored care anesthesia, which is included in the above indication, has been increased to $1.0 \mu \mathrm{g} / \mathrm{kg} /$ hour.
Correspondence: Takayuki Kunisawa Department of Anesthesiology and Critical Care Medicine, Asahikawa Medical University, 2-I-I-I Midorigaokahigashi, Asahikawa, Hokkaido 07885 I0, Japan

Tel +8 I | 66682583

Fax $+8 I I 66682589$

Email taka.kunisawa@nifty.ne.jp 

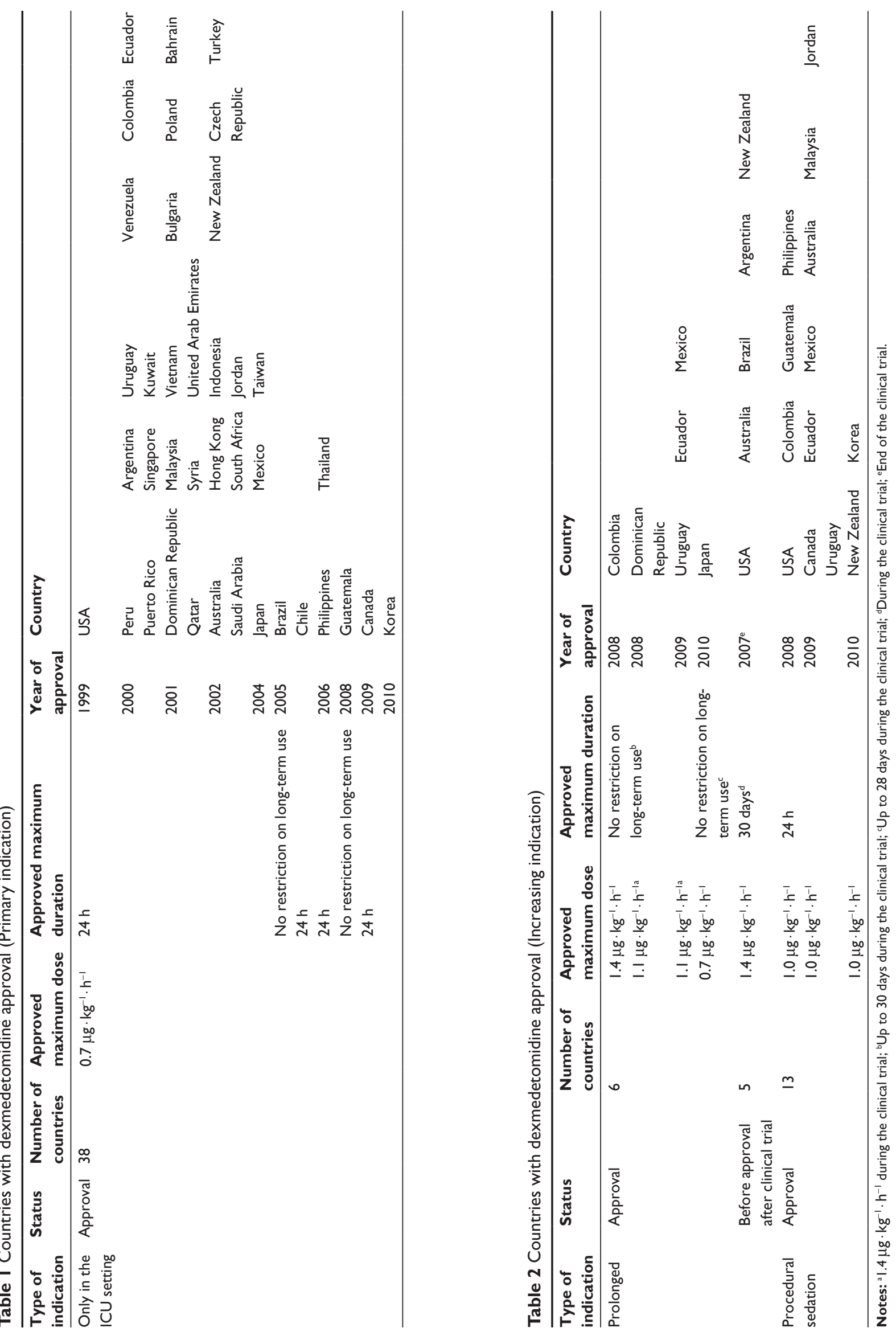
Long-term administration has been approved in five countries since the first approval was acquired in Colombia in 2008. In 2010, Japan became the sixth country to grant approval for long-term administration of dexmedetomidine. This review focuses on the prolonged use of dexmedetomidine, which is one of the two expanding indications for this sedative, and discusses the effectiveness and safety of its prolonged use.

\section{Treatment duration}

In the Japanese package insert, ${ }^{3}$ the following instructions are provided with regard to prolonged dexmedetomidine administration: "Dexmedetomidine can be administered during intubation or during and after intubation, however strict monitoring for systemic condition of patients should be continued if duration exceeds 5 days because of lack of experience of administration over 5 days". A Phase III clinical trial was performed in Japan in 2007-2008 to evaluate the safety and efficacy of long-term dexmedetomidine in patients requiring 24 hours or more of sedation in the intensive care unit. This study examined a protocol in which dexmedetomidine was used for up to 28 days. In this trial, dexmedetomidine was administered for a mean duration of $88 \pm 98$ hours, ${ }^{4}$ and the maximum duration of dexmedetomidine administration was 478 hours.

Riker et $\mathrm{al}^{5}$ reported a multicenter trial in which the safety and efficacy of dexmedetomidine was compared with that of midazolam in critically ill, mechanically ventilated patients in five countries (US, Australia, Brazil, Argentina, and New Zealand). The protocol involved dexmedetomidine administration for up to 30 days. In that study, 244 patients were treated with dexmedetomidine for a median duration of 3.5 days (interquartile range [IQR]: 2.0-5.2 days). A retrospective cohort study by Wunsch et $\mathrm{al}^{6}$ examined 58,391 patients who received intravenous infusion sedation; 2535 of them received dexmedetomidine. The mean duration of dexmedetomidine infusion ( \pm standard deviation [SD]) was $1.5 \pm 2.0$ days, and $31.5 \%$ of the patients received dexmedetomidine infusion for more than 1 day.

Venn et $\mathrm{al}^{7}$ performed a pilot study in 12 patients in a medical intensive care unit in the UK, and Shehabi et $\mathrm{al}^{8}$ performed a pilot study in 20 patients in both medical and surgical intensive care units in Australia. In both studies, dexmedetomidine was used if required for up to 7 days. In a study by Wunsch et al, five of 12 patients received dexmedetomidine infusion for more than 24 hours, and one patient received dexmedetomidine for a maximum duration of 72 hours. In the studies by Venn et al and Shehabi et al, all 20 patients received dexmedetomidine for more than 24 hours, and one patient received dexmedetomidine for 7 days.

The protocol in a multicenter, prospective, randomized, double-blind, double-dummy, active comparator study performed by Ruokonen et $\mathrm{al}^{9}$ in Finland and Switzerland allowed dexmedetomidine to be administered for up to 14 days, and 41 patients received dexmedetomidine for a median duration of 40 (range 3-198) hours.

Pandharipande et $\mathrm{l}^{10,11}$ conducted two controlled studies to compare the effects of dexmedetomidine with those of lorazepam on acute brain dysfunction in mechanically ventilated patients and to compare the effects of dexmedetomidine with those of lorazepam on clinical outcome in patients with or without sepsis. In these studies, dexmedetomidine was administered, if required, for up to 5 days.

\section{Dosing}

The doses used in the aforementioned studies were used to evaluate the efficacy of long-term administration of dexmedetomidine. The study conducted by Venn et $\mathrm{al}^{7}$ was a small, uncontrolled study involving 12 patients and showed an interesting change in protocol and results. Their dosing protocol for long-term administration had been a maintenance dose of $0.2-0.7 \mu \mathrm{g} / \mathrm{kg} /$ hour with a loading dose of $1.0 \mu \mathrm{g} / \mathrm{kg}$ over 10 minutes. However, the protocol was revised, and the maximum maintenance dose was increased to $2.5 \mu \mathrm{g} / \mathrm{kg} / \mathrm{hour}$ from the fifth patient onwards because sedation goals were not achieved with the earlier dose in the first four patients. The mean dexmedetomidine infusion rate in the last eight patients was $1.0 \pm 0.7 \mu \mathrm{g} / \mathrm{kg} /$ hour, and the median value of the maximum dose in the last eight patients was 1.35 (range 0.4-2.5) $\mu \mathrm{g} / \mathrm{kg} /$ hour. The authors stated that medical patients might need higher infusion rates of dexmedetomidine.

In the study by Shehabi et $a{ }^{8}{ }^{8} 20$ critically ill patients received dexmedetomidine at a dose of $0.2-0.7 \mu \mathrm{g} / \mathrm{kg} / \mathrm{hour}$, but a loading dose of dexmedetomidine was omitted because the patients had already received other sedative drugs, which were administered until 1 hour after administration of dexmedetomidine was started. Most patients had an acceptable quality of sedation, ie, $83 \%$ of the patients had sedation levels between 2 and 5 on the Ramsay Sedation Scale during the study period, with minimal or no additional midazolam administration (median 4 , range $0.5-10 \mathrm{mg} /$ day) in 16 patients and minimal or no additional analgesia (median 2, range 0.5-4.5 mg/day for morphine; median 55, range $14-63 \mu \mathrm{g} /$ day for fentanyl) in ten patients. However, the authors suggested that some patients could have benefited from a higher dose, because high doses of midazolam were 
required to facilitate the management of a deteriorating clinical state in four patients.

Two randomized controlled trials performed by Pandharipande et $\mathrm{al}^{10,11}$ comparing the efficacy of dexmedetomidine with that of lorazepam used a protocol that permitted dexmedetomidine administration at doses up to $1.5 \mu \mathrm{g} / \mathrm{kg} /$ hour. In the latter study, the median infusion rate for dexmedetomidine was 0.74 (IQR: 0.39-1.04) $\mu \mathrm{g} / \mathrm{kg} / \mathrm{hour}$; the median dexmedetomidine dose given to patients who had sepsis was 0.8 (IQR: $0.3-1.1$ ) $\mu \mathrm{g} / \mathrm{kg} /$ hour, and the median dose administered to patients who did not have sepsis was $0.6 \mu \mathrm{g} / \mathrm{kg} /$ hour. Ruokonen et $\mathrm{al}^{9}$ adjusted dexmedetomidine stepwise at five doses $(0.25,0.5,0.8,1.1$, and $1.4 \mu \mathrm{g} / \mathrm{kg} / \mathrm{hour})$ after infusion of a dose of $0.8 \mu \mathrm{g} / \mathrm{kg} /$ hour for 1 hour without an initial dose, and the patients in their study received a median dexmedetomidine dose of 0.8 (range $0.3-1.4$ ) $\mu \mathrm{g} / \mathrm{kg} /$ hour.

Ricker et $\mathrm{al}^{5}$ adopted a strategy using doses up to $1.4 \mu \mathrm{g} / \mathrm{kg} /$ hour, which was twice the limit approved at that time by the FDA. The mean \pm SD maintenance infusion dose of dexmedetomidine was $0.83 \pm 0.37 \mu \mathrm{g} / \mathrm{kg} /$ hour. The dexmedetomidine dose was $0.2-0.7 \mu \mathrm{g} / \mathrm{kg} /$ hour in 95 of 244 patients (39\%), $0.71-1.1 \mu \mathrm{g} / \mathrm{kg} /$ hour in 78 of 244 patients (32\%), and more than $1.1 \mu \mathrm{g} / \mathrm{kg} /$ hour in 71 of 244 patients (29\%). A Phase III clinical trial performed in Japan used the maximum dose of $0.7 \mu \mathrm{g} / \mathrm{kg} /$ hour. However, the actual administered infusion rate has not been reported. ${ }^{4}$

\section{Efficacy}

\section{Sedation}

Riker et $\mathrm{al}^{5}$ confirmed that there was no difference in the primary sedation efficacy achieved by dexmedetomidine or midazolam. The percentage of the duration for which patients were noted to be within the target Richmond Agitation-Sedation Score (RASS) range was $77.3 \%$ for those treated with dexmedetomidine and $75.1 \%$ for those treated with midazolam (difference $2.2 \%, 95 \%$ confidence interval $[\mathrm{CI}]:-3.2 \%-7.5 \% ; P=0.18)$.

Pandharipande et $\mathrm{al}^{10}$ revealed that, in comparison with patients sedated with lorazepam, those sedated with dexmedetomidine spent more time at the level of sedation targeted by both nurses and physicians. The median percentages of RASS scores in the patients treated with dexmedetomidine that were within one point of the nurse and physician goal were 80 (IQR: 58-100) and 67 (IQR: 50-85), respectively, and these scores were higher than the corresponding values in the lorazepam group (67\% [48\%-83\%] and 55\% [8\%-67\%], respectively). The percentage of days on which the RASS scores deviated by $\geq 2$ points from the nurse goal in the case of patients treated with dexmedetomidine was lower than that in the lorazepam group (median 15\% [IQR: 0\%-33\%] vs $33 \%$ [IQR: $11 \%-48 \%]$ ). The number of days patients were noted to be oversedated was lower in the dexmedetomidine group than in the lorazepam group (median 1 [IQR: 0-2.2] vs 2 [IQR: 1-3.5]).

In the study reported by Pandharipande et al, ${ }^{11}$ the efficiency of sedation in the patients who did not have sepsis was similar for both treatment groups $(67 \%$ of days [IQR: $50 \%-86 \%$ ] vs $60 \%$ of days [IQR: $27 \%-75 \%$ ]; $P=0.27$ ). However, among the patients with sepsis, those sedated with dexmedetomidine achieved sedation within one point of the RASS target more often than those sedated with lorazepam (accurate sedation on $67 \%$ of days [IQR: $50 \%-83 \%$ ] vs $52 \%$ of days [IQR: $0 \%-67 \%$ ]; $P=0.01$ ).

The study conducted by Ruokonen et al, ${ }^{9}$ in which the efficacy of dexmedetomidine was compared with that of standard care using either propofol or midazolam for sedation, did not confirm the noninferiority of dexmedetomidine in comparison with standard care. The target RASS level was reached in a median of $64 \%$ (dexmedetomidine) and 63\% (standard care) for sedation time (not statistically significant). For patients with an RASS target level of -3 to 0 (dexmedetomidine $78 \%$; standard care $80 \%$ ), the target sedation level was achieved in $74 \%$ of cases when dexmedetomidine was administered and in $64 \%$ of cases when standard care was administered (not statistically significant), whereas for patients with an RASS target level $\leq 4$, the target sedation level was achieved in $42 \%$ of cases when dexmedetomidine was administered and in $62 \%$ of cases when standard care was administered $(P=0.006)$; these results suggested the unsuitability of dexmedetomidine for deep sedation.

\section{Delirium and coma}

Riker et $\mathrm{al}^{5}$ demonstrated the superiority of dexmedetomidine with regard to the treatment or prevalence of delirium. During this study, the effect of dexmedetomidine treatment on delirium, as measured by a generalized estimating equation, was a $24.9 \%$ reduction ( $95 \%$ CI: $16 \%-34 \% ; P<0.001$ ). The prevalence of delirium was $54 \%(132 / 244)$ in patients treated with dexmedetomidine, in contrast with a prevalence of $76.6 \%(93 / 122)$ in patients treated with midazolam (difference $22.6 \%$, 95\% CI: $14 \%-33 \%$; $P<0.001$ ). Moreover, despite the shorter duration of study drug treatment (dexmedetomidine vs midazolam, median [IQR] 3.5 [2.0-5.2] vs 4.1 [2.8-6.1]; $P=0.01$ ), the number of delirium-free days was greater for patients treated with dexmedetomidine ( 2.5 days vs 1.7 days; $P=0.002$ ). In the study by Pandharipande et al, ${ }^{10}$ 
there were no intergroup differences in the duration of delirium-free days (median [IQR] 9 [5-11] vs 7 [5-10]; $P=0.09$ ) or the prevalence of delirium (number [\%], 41 [79\%] vs 42 [82\%]; $P=0.65)$. However, because there were significant intergroup differences in the duration of coma-free days (median [IQR] 10 [9-12] vs 8 [5-10]; $P<0.001$ ) and prevalence of coma (number [\%], 33 [63\%] vs 47 [92\%]; $P<0.001$ ), sedation with dexmedetomidine resulted in a higher duration of survival without delirium or coma (median days [IQR], 3.0 [1-6] vs $7.0[1-10] ; P=0.01)$ and a lower prevalence of coma $(n=45[63 \%])$ than with sedation using lorazepam $(\mathrm{n}=47$ [92\%]; $P<0.001)$.

Another study conducted by Pandharipande et $\mathrm{al}^{11}$ showed that patients with sepsis who were treated with dexmedetomidine had 3.2 more delirium-free/coma-free days on average (95\% CI for difference: 1.1-4.9 days). Moreover, among all patients (including patients who did not have sepsis), those sedated with dexmedetomidine had a $70 \%$ lower likelihood of having delirium on any given day in comparison with patients sedated with lorazepam $(P$ for treatment $=0.004)$.

A pilot study by Ruokonen et $\mathrm{al}^{9}$ showed that delirium was more common in patients treated with dexmedetomidine (43.9\% vs $25.0 \%$ for standard care; $P=0.035$ ) when analyzed as the combined endpoint of the confusion assessment method for the intensive care unit (CAM-ICU) and adverse events of delirium and confusion. However, more CAM-ICU assessments were performed in the dexmedetomidine-treated group (106 for dexmedetomidine vs 84 for standard care), and the proportion of positive CAM-ICU results was comparable ( $17.0 \%$ for dexmedetomidine vs $17.9 \%$ for standard care, not statistically significant).

\section{Clinical outcome}

Riker et $\mathrm{al}^{5}$ showed that the time to extubation in patients treated with dexmedetomidine was 1.9 days shorter than that in patients treated with midazolam (3.7 days [95\% CI: 3.1-4.0 days] vs 5.6 days [95\% CI: 4.6-5.9 days], respectively; $P=0.01)$. However, the median length of intensive care stay was similar (5.9 days [95\% CI: 5.7-7.0 days] vs 7.6 days [95\% CI: $6.7-8.6$ days]; $P=0.24$ ), although the length of stay for the dexmedetomidine-treated group tended to be shorter than that for the midazolam-treated group. There was no intergroup difference in 30-day mortality following intensive care admission between patients treated with dexmedetomidine $(22.5 \%$ [55/244]) and those treated with midazolam $(25.4 \%$ [31/122]; $P=0.60)$, and no death was considered to be related to dexmedetomidine. The percentage of patients transferred alive from the intensive care unit was also similar in both groups, (81.5\% [199/244] for patients treated with dexmedetomidine vs $81.9 \%$ [100/122] for patients treated with midazolam; $P>0.99$ ).

In one of the studies conducted by Pandharipande et al, ${ }^{10}$ no significant differences were noted between the dexmedetomidine-treated and lorazepam-treated groups for ventilator-free days ( 22 vs 18 days; $P=0.22$ ), length of stay in the intensive care unit ( 7.5 vs 9 days; $P=0.92$ ), and rate of mortality after 28 days $(17 \%$ vs $27 \% ; P=0.18)$. Moreover, the 12-month time to death in the dexmedetomidine-treated group and the lorazepam-treated group was 363 and 188 days, respectively. The likelihood of dying at 12 months was similar between the groups (hazards ratio [HR]: $0.8 ; 95 \%$ CI: $0.5-1.4 ; P=0.48)$.

The other study conducted by Pandharipande et $\mathrm{al}^{11}$ showed that patients with sepsis who were sedated with dexmedetomidine had a mean of 6.0 (95\% CI: 0.3-11.0 days) more ventilator-free days than did patients receiving lorazepam. Interestingly, patients with sepsis who were sedated with dexmedetomidine also had a lower risk of death at 28 days than those sedated with lorazepam (HR: 0.3, 95\% CI: 0.1-0.9); this beneficial effect was not seen in patients who did not have sepsis (HR: 4.0, 95\% CI: 0.4-35.5; $P$ for interaction $=0.11$ ).

In the study by Ruokonen et al, ${ }^{9}$ there were no significant differences between the dexmedetomidine and standard care groups in terms of duration for which mechanical ventilation was needed, time required for weaning, number of ventilatorfree days, or time to discharge from the hospital. After post hoc adjustment, the duration of mechanical ventilation was shorter in patients treated with dexmedetomidine (77.2 [range: 17.5-338.8] hours vs 110.6 [range: 20.1-675.0] hours; $P=0.025)$, and median duration of mechanical ventilation in patients with light-to-moderate sedation in the dexmedetomidine-treated group was shorter than that in the standard care group (70.2 [range: 17.5-225.4] hours vs 93.7 [range: 20.1-675.0] hours; $P=0.027$ ).

\section{Adverse events}

The study conducted by Riker et $\mathrm{al}^{5}$ showed that the number of dexmedetomidine-treated patients who developed treatment-related adverse events was greater than the number of midazolam-treated patients who developed these events (40.6\% [99/244] vs 28.7\% [35/122]; $P=0.03)$, primarily because of a greater incidence of bradycardia $(42.2 \%$ [103/244] vs $18.9 \%$ [23/122]; $P<0.001)$. In contrast, the incidence of tachycardia in patients treated with midazolam was higher than that seen in patients treated with dexmedetomidine (44.3\% [54/122] vs 25.4\% [62/244]; $P<0.001)$. There 
were no significant intergroup differences in the incidences of hypotension or hypertension. Regarding adverse events that required intervention, only the incidence of hypertension had a significant intergroup difference (midazolam 29.5\% [36/122] vs dexmedetomidine 18.9\% [46/244]), although the incidence of bradycardia that required intervention was higher in patients treated with dexmedetomidine than in patients treated with midazolam.

Ruokonen et $\mathrm{al}^{9}$ showed that serious adverse events were equally common. Seventeen patients (42\%) treated with dexmedetomidine had serious adverse events (including three patients with bradycardia and two patients with hypotension), and 18 patients (41\%) treated with midazolam or propofol had serious adverse events (including two patients with cardiac failure and one patient with hypotension). There were no intergroup differences in the incidence of serious adverse events.

Pandharipande et $\mathrm{al}^{11}$ showed that there were no significant intergroup differences in hemodynamic variables for patients with sepsis. In patients who did not have sepsis, the only significant intergroup difference was for the incidence of sinus bradycardia (24\% for dexmedetomidine vs $0 \%$ for lorazepam; $P=0.02$ ). Another study conducted by Pandharipande et $\mathrm{al}^{10}$ showed that patients in the dexmedetomidine-treated and lorazepam-treated groups had comparable blood pressure, vasoactive drug usage, and incidence of tachycardia. Patients in the dexmedetomidine-treated group had a higher incidence of sinus bradycardia (heart rate $<60$ beats per minute) than those in the lorazepam-treated group, although one patient from each group had an episode of heart rate lower than 40 beats per minute.

\section{Rebound complications and/or withdrawal events}

Riker et $\mathrm{al}^{5}$ reported that rebound hypertension and tachycardia did not occur following abrupt discontinuation of dexmedetomidine infusion. Ruokonen et $\mathrm{al}^{9}$ observed no signs of cardiovascular instability and/or rebound after cessation of sedation with dexmedetomidine. Venn et $\mathrm{al}^{7}$ observed that following discontinuation of dexmedetomidine, there were small, but sustained increases in systolic and diastolic arterial pressure and heart rate, but there were no clinically important rebound phenomena.

In the study by Shehabi et al, ${ }^{8}$ the incidences of these phenomena were recorded for 24 hours after abrupt cessation of the study drug, and minimal changes were observed. A maximum mean systolic blood pressure value of $154 \pm 24.7 \mathrm{mmHg}$ ( $7 \%$ increase) from a baseline mean of $143 \pm 24 \mathrm{mmHg}$ was recorded 5 hours after cessation. The mean baseline heart rate at cessation of dexmedetomidine administration was $86 \pm 27$ beats per minute, and a maximum mean heart rate of $97 \pm 27$ beats per minute (11\% increase) was observed after 14 hours. These findings indicated no evidence of cardiovascular rebound 24 hours after abrupt cessation of infusion.

Riker et $\mathrm{al}^{5}$ reported rare drug-related withdrawal events, such as agitation, headache, hyperhidrosis, nausea, nervousness, tremor, or vomiting after stopping the study drug. Overall, 4.9\% (12/244) of dexmedetomidine-treated patients and $8.2 \%(10 / 122)$ of midazolam-treated patients experienced at least one event related to withdrawal within 24 hours of discontinuing the study drug $(P=0.25)$.

\section{Other outcomes}

There were two interesting outcomes in the study by Riker et al. ${ }^{5}$ Hyperglycemia occurred more frequently among dexmedetomidine-treated patients. The rates of treatment with corticosteroids were similar (65.5\% [160/244] in dexmedetomidine-treated patients vs $68.9 \%$ [84/122] in midazolam-treated patients), as was the use of insulin therapy (77.8\% [190/244] of dexmedetomidine-treated patients vs $74.8 \%$ [91/122] in midazolam-treated patients); however, the reasons for these results are not known. The incidence of infection occurring during the double-blind period was lower in dexmedetomidine-treated patients $(10.2 \%$ [25/244] vs $19.7 \%$ [24/122] in midazolam-treated patients; $P=0.02)$. This included lower rates of urinary tract infections $(0 \%$ in dexmedetomidine-treated patients vs 3.3\% [4/122] in midazolam-treated patients; $P=0.02$ ) and hospital-acquired pneumonia $(1.2 \%$ [3/244] in dexmedetomidine-treated patients vs $4.9 \%$ [6/122] in midazolam-treated patients; $P=0.07)$. The results may be because use of dexmedetomidine reduced the time needed for mechanical ventilation and reduced the length of intensive case stay. However, in the study conducted by Pandharipande et al, the development of new secondary infections beyond the first 48 hours after enrollment was similar in the patients without sepsis in the dexmedetomidine and lorazepam study arms (17\% vs $15 \%) .{ }^{11}$

\section{Cost}

Dasta et $\mathrm{al}^{12}$ performed a secondary analysis of a previously published study conducted by Riker et $\mathrm{al}^{5}$ to analyze intensive care unit cost differences in patients after randomization to dexmedetomidine or midazolam for sedation. The unadjusted median total intensive care unit cost was significantly lower for patients in the dexmedetomidine-treated group (US\$27,694; $P<0.025)$ in comparison with US\$34,122 in the midazolamtreated group. The median costs calculated after adjusting 
for censored intensive care unit and ventilation times were about $50 \%$ higher in each group and remained significantly lower in the dexmedetomidine-treated group. In the primary analysis, after adjustment for covariates and censoring patients, the dexmedetomidine-treated group achieved a median cost saving of US\$9679 (95\% CI: US\$2314US $\$ 17,045 ; P<0.01$ for intensive care unit). After adjusting for covariates and censoring patients, dexmedetomidine use resulted in significant median savings in both intensive care unit costs (US\$6584, 95\% CI: US\$727-US\$12,440) and the component cost of mechanical ventilation (US\$2958, 95\% CI: US\$698-US\$5219). The median component costs associated with treating adverse drug reactions were also significantly lower in the dexmedetomidine-treated group (US\$229, 95\% CI, US\$49-US\$409; $P<0.013$ ). These cost savings were observed despite the higher study drug acquisition cost for dexmedetomidine (mean cost US\$1826 vs US\$80 for midazolam; median cost US\$1166 vs US\$60 for midazolam).

Pandharipande et $\mathrm{al}^{10}$ demonstrated that the median total hospital cost in their dexmedetomidine-treated group was approximately US\$22,500 higher than that in their lorazepam-treated group; however, the difference was not statistically significant. They concluded that despite the higher cost of dexmedetomidine, the benefits of sustained sedation with this agent were realized, with comparable overall pharmacy, respiratory, intensive care unit, and hospital costs.

\section{Pediatric patients}

There are no large, controlled, prospective or randomized studies of dexmedetomidine in pediatric patients, and there are no studies comparing dexmedetomidine with placebo or other sedative drugs, such as midazolam or propofol, in this age group.

One prospective observational study was reported by Buck and Wilson, ${ }^{13}$ in which 17 patients received dexmedetomidine for dose reduction of other sedatives that could cause respiratory depression, for use as an alternative during prolonged intubation after other sedatives failed to achieve adequate sedation, or for treating patients who developed tolerance to other sedatives although they received midazolam or an opioid before or during administration of dexmedetomidine.

Four retrospective medical chart reviews ${ }^{14-17}$ have been published presenting experiences with prolonged dexmedetomidine infusion in infants and children. All of them indicated the efficacy of prolonged dexmedetomidine use. However, in these studies, dexmedetomidine was not used as a first-choice sedative; rather, it was added to an existing sedative regimen to facilitate dose reduction of the standard sedation agents and extubation. Some indications and dose recommendations were introduced, and their efficacy and safety were investigated.

\section{Dosing and duration}

In the study by Buck and Wilson, ${ }^{13}$ dexmedetomidine was administered without a loading dose to all patients (mean age, $31.4 \pm 60.9$ months; median age, 5 months [range 1 month to 17 years]) at a starting dose of $0.2 \pm 0.2$ (range, $0.1-0.5) \mu \mathrm{g} / \mathrm{kg} /$ hour. The mean $\pm \mathrm{SD}$ duration of therapy was $32 \pm 21$ (range 3-75) hours. The duration of therapy exceeded the 24 -hour limit in $50 \%$ of cases. Bejian et $\mathrm{l}^{15}$ identified 54 patients who received dexmedetomidine; the median age was 6 months (range 1 day to 16 years). The initial and maximum doses of dexmedetomidine were $0.38 \pm 0.13$ (range $0.2-0.7$ ) $\mu \mathrm{g} / \mathrm{kg} /$ hour and $0.8 \pm 0.48$ (range 0.3-2.0) $\mu \mathrm{g} / \mathrm{kg} / \mathrm{hour}$, respectively, and the infusion duration was $37.3 \pm 37.4$ (range 2-177) hours.

The chart reviews of 65 burns patients with a mean age of 5 (range $0.6-17$ ) years performed by Walker et $\mathrm{al}^{16}$ revealed that for patients receiving dexmedetomidine at the initial dose of $0.2 \mu \mathrm{g} / \mathrm{kg} /$ hour with a loading dose of $1 \mu \mathrm{g} / \mathrm{kg}$ (in $40 \%$ of patients) or no loading dose (in $60 \%$ of the patients), the average duration of dexmedetomidine infusion was 11 (range 2-50) days, with a mean dose of 0.5 (range $0.1-2.0) \mu \mathrm{g} / \mathrm{kg} / \mathrm{hour}$.

In the study performed by Carroll et al, ${ }^{16}$ dexmedetomidine was administered 74 times to 60 children. The median age of the children was 1.5 (range $0.1-17.2$ ) years. The median dose required to maintain adequate sedation was 0.7 (range $0.2-2.5) \mu \mathrm{g} / \mathrm{kg} /$ hour after no loading dose, with a median duration of therapy of 23 (range 3-451) hours. Reiter et $\mathrm{al}^{14}$ reported that eight of 29 patients (aged $5.3 \pm 6.1$ years) received a loading dose of dexmedetomidine $0.5-1.0 \mu \mathrm{g} / \mathrm{kg}$ prior to the start of the infusion. Dexmedetomidine was initiated at $0.36 \pm 0.16$ (range $0.1-0.75 \mu \mathrm{g} / \mathrm{kg} / \mathrm{hour}$ ), and the maximum dose was $0.65 \pm 0.34$ (range $0.2-1.5$ ) $\mu \mathrm{g} / \mathrm{kg} /$ hour. The mean duration of dexmedetomidine therapy was $110 \pm 83$ (range 32-378, median 76) hours.

\section{Efficacy}

There are no studies in which the efficacy of dexmedetomidine has been compared with that of other agents in pediatric patients, because dexmedetomidine was initiated as an adjunctive and/or alternative to conventional sedation. Moreover, because validated sedation scales were not used to assess the efficacy of sedation nor to set or assess 
the ability to achieve sedation goals, conclusions cannot be drawn regarding the efficacy of dexmedetomidine in achieving sedation goals in pediatric patients. The objectives of dexmedetomidine administration were: reduction in the doses of benzodiazepines and/or opioids to reduce the risk of respiratory depression during extubation: use as an alternative sedative agent in patients who did not achieve adequate sedation or those who become paradoxically agitated with traditional sedatives; and sedation in nonintubated children to provide a titratable level of sedation without respiratory depression. Therefore, the efficacy of dexmedetomidine has mainly been investigated for its effectiveness in reducing the use of conventional sedation agents.

Buck and Wilson ${ }^{13}$ reported that use of dexmedetomidine allowed discontinuation of midazolam or reduction in dose of midazolam prior to extubation in 13 of 14 cases, although discontinuation of opioid analgesics was not attempted. The investigation by Bejian et $\mathrm{al}^{15}$ revealed that patients given dexmedetomidine received significantly less fentanyl and midazolam than controls. The mean total doses of fentanyl $(16.6 \pm 4.2 \mu \mathrm{g} / \mathrm{kg} /$ day $)$ and midazolam $(0.26 \pm 0.1 \mu \mathrm{g} / \mathrm{kg} /$ day $)$ were lower than the corresponding values in the control group (fentanyl, $47.5 \pm 15.1 \mu \mathrm{g} / \mathrm{kg} / \mathrm{day}, P=0.014$; midazolam, $1.08 \pm 0.47 \mu \mathrm{g} / \mathrm{kg} / \mathrm{day}, P=0.006$ ), although the durations of fentanyl and midazolam infusions were not significantly different between the groups.

Walker et al ${ }^{15}$ noted that initiation of dexmedetomidine could change the sedation rating from inadequate sedation to adequate sedation in all their 65 pediatric burns patients. Carroll et al ${ }^{17}$ found that use of dexmedetomidine to facilitate weaning from other sedative agents could reduce the use of fentanyl infusion ( $43 \%$ vs $17 \% ; P=0.009$ ) and scheduled lorazepam infusion ( $30 \%$ vs $10 \% ; P=0.02)$. Reiter et al ${ }^{14}$ showed that the overall degree of sedation (continuous sedation, pulse as-needed sedation) was generally reduced during dexmedetomidine therapy, although the number of asneeded doses was higher during dexmedetomidine therapy, compared with the number of as-needed doses before and after therapy.

\section{Safety}

Buck and Wilson ${ }^{13}$ revealed that mean arterial pressures before and after starting dexmedetomidine were not significantly different $(P=0.76)$, nor were the mean arterial pressure values at $1(P=0.31)$ or 12 hours after discontinuation $(P=0.29)$ significantly different from those before discontinuation. No significant differences were noted in heart rate at the start of therapy $(P=0.09)$, at the discontinuation of therapy
( $P=0.06)$, or 12 hours later $(P=0.17)$. Hypotension occurred during treatment in one patient who had sepsis. However, this effect was considered to be caused by sepsis itself because the hypotension continued after dexmedetomidine was discontinued, and blood pressure improved with administration of antibiotics and supportive therapy.

Bejian et $\mathrm{al}^{15}$ found that mean heart rates after discontinuation of infusion were statistically lower than those prior to or during the infusion, ie, 120 and 124 beats per minute (15 minutes before and 2 hours after discontinuation, respectively) vs 138 and 133 beats per minute ( 2 hours before and 2 hours after initiation), respectively $(P<0.05)$. Respiratory rates 15 minutes and 2 hours after discontinuation were statistically higher than those before infusion and during infusion, ie, 29 breaths per minute vs 24 breaths per minute $(P<0.05)$. However, they concluded that there were no clinically significant changes in the physiological parameters associated with initiation, maintenance, or discontinuation of dexmedetomidine infusion, because no intervention was required for the abovementioned change in parameters and because initiation, maintenance, or discontinuation of dexmedetomidine did not cause any significant changes in other physiological parameters.

Walker et $\mathrm{al}^{16}$ reported two deaths and one episode of hypotension requiring epinephrine infusion. However, they felt that these episodes were related to the severity of the patients' illnesses and not to administration of dexmedetomidine. They did not find clinically significant episodes of bradycardia or hypertension. Rebound hypertension or withdrawal was not observed and tachyphylaxis was not noted. The patients' blood glucose levels averaged $121.2 \pm 8.9 \mathrm{mg} / \mathrm{dL}$ while on dexmedetomidine infusion and $117.1 \pm 12.1 \mathrm{mg} / \mathrm{dL}$ while off it, and the difference was not significant. Carroll et a $1{ }^{17}$ reported that $20 \%$ of their pediatric patients receiving dexmedetomidine experienced adverse effects, ie, hypotension (9\%), hypertension (8\%), and bradycardia (3\%). Almost all of these events resolved without treatment or withholding dexmedetomidine infusion; one patient required fluid therapy for treatment of hypotension. Reiter et $\mathrm{al}^{14}$ found that initiation of therapy with dexmedetomidine was associated with a transient yet statistically significant reduction in heart rate (from $120 \pm 28$ beats per minute to $107 \pm 27$ beats per minute; $P=0.002$ ), but without a change in blood pressure.

\section{Conclusion}

Dexmedetomidine can be an alternative long-term sedative for achieving sedation goals. Dexmedetomidine offers the 
major advantage of a reduction in the incidence of delirium and coma during long-term sedation in the intensive care unit setting. Adverse effects such as bradycardia that occur during short-term sedation with dexmedetomidine are unavoidable. Interestingly, a secondary beneficial effect associated with the use of dexmedetomidine is a reduction in the incidence of infection; this appears to be because the use of dexmedetomidine reduces intensive care unit stay and/or duration of mechanical ventilation. Rebound and/or withdrawal effects after discontinuation at the end of prolonged sedation do not seem to be a concern.

In pediatric patients, a prospective study is awaited for confirmation of the efficacy and safety of dexmedetomidine. However, dexmedetomidine can be expected to serve as an adding agent and/or alternative for dose reduction of traditional agents to minimize the respiratory effects of extubation and as an alternative in patients who are not comfortable with receiving conventional agents. Dexmedetomidine has potential as a main drug for both long- and short-term sedation, and the findings of further studies are awaited with interest.

\section{Disclosure}

The author reports no conflicts of interest in this work.

\section{References}

1. Ebert TJ, Hall JE, Barney JA, Uhrich TD, Colinco MD. The effects of increasing plasma concentrations of dexmedetomidine in humans. Anesthesiology. 2000;93(2):382-394.

2. Hall JE, Uhrich TD, Barney JA, Arain SR, Ebert TJ. Sedative, amnestic, and analgesic properties of small-dose dexmedetomidine infusions. Anesth Analg. 2000;90(3):699-705.

3. Precedex ${ }^{\circledR}$ [Package insert]. Osaka, Japan: Hospira Japan Co Ltd; 2010.
4. Taiji K. Phase III non-blinded long-term treatment study to evaluate the safety and efficacy of long-term treatment with dexmedetomidine. Hospira Japan in-house clinical study report; 2009.

5. Riker RR, Shehabi Y, Bokesch PM, et al. Dexmedetomidine vs midazolam for sedation of critically ill patients: A randomized trial. JAMA. 2009;301(5):489-499.

6. Wunsch H, Kahn JM, Kramer AA, et al. Dexmedetomidine in the care of critically ill patients from 2001 to 2007: An observational cohort study. Anesthesiology. 2010;113(2):386-394.

7. Venn M, Newman J, Grounds M. A Phase II study to evaluate the efficacy of dexmedetomidine for sedation in the medical intensive care unit. Intensive Care Med. 2003;29(2):201-207.

8. Shehabi Y, Ruettimann U, Adamson H, Innes R, Ickeringill M Dexmedetomidine infusion for more than 24 hours in critically ill patients: Sedative and cardiovascular effects. Intensive Care Med. 2004; 30(12):2188-2196.

9. Ruokonen E, Parviainen I, Jakob SM, et al. Dexmedetomidine versus propofol/midazolam for long-term sedation during mechanical ventilation. Intensive Care Med. 2009;35(2):282-290.

10. Pandharipande PP, Pun BT, Herr DL, et al. Effect of sedation with dexmedetomidine vs lorazepam on acute brain dysfunction in mechanically ventilated patients: The MENDS randomized controlled trial. JAMA. 2007;298(22):2644-2653.

11. Pandharipande PP, Sanders RD, Girard TD, et al. Effect of dexmedetomidine versus lorazepam on outcome in patients with sepsis: An a priori-designed analysis of the MENDS randomized controlled trial. Crit Care. 2010;14(2):R38.

12. Dasta JF, Kane-Gill SL, Pencina M, et al. A cost-minimization analysis of dexmedetomidine compared with midazolam for long-term sedation in the intensive care unit. Crit Care Med. 2010;38(2):497-503.

13. Buck ML, Wilson DF. Use of dexmedetomidine in the pediatric intensive care unit. Pharmacotherapy. 2008;28(1):51-57.

14. Reiter PD, Pietras M, Dobyns EL. Prolonged dexmedetomidine infusions in critically ill infants and children. Indian Pediatr. 2009; 46(9):767-773.

15. Bejian S, Valasek C, Nigro JJ, Cleveland DC, Willis BC. Prolonged use of dexmedetomidine in the paediatric cardiothoracic intensive care unit. Cardiol Young. 2009;19(1):98-104.

16. Walker J, MacCallum M, Fischer C, Kopcha R, Saylors R, McCall J. Sedation using dexmedetomidine in pediatric burn patients. J Burn Care Res. 2006;27(2):206-210.

17. Carroll CL, Krieger D, Campbell M, Fisher DG, Comeau LL, Zucker AR. Use of dexmedetomidine for sedation of children hospitalized in the intensive care unit. J Hosp Med. 2008;3(2):142-147.
Therapeutics and Clinical Risk Management

\section{Publish your work in this journal}

Therapeutics and Clinical Risk Management is an international, peerreviewed journal of clinical therapeutics and risk management, focusing on concise rapid reporting of clinical studies in all therapeutic areas, outcomes, safety, and programs for the effective, safe, and sustained use of medicines. This journal is indexed on PubMed Central, CAS,

\section{Dovepress}

EMBase, Scopus and the Elsevier Bibliographic databases. The manuscript management system is completely online and includes a very quick and fair peer-review system, which is all easy to use. Visit http://www.dovepress.com/testimonials.php to read real quotes from published authors. 